\title{
MolView Users Guide
}

by

Brian P. Walenz

\section{REOEIVE \\ AUG 261996 \\ OSTI}

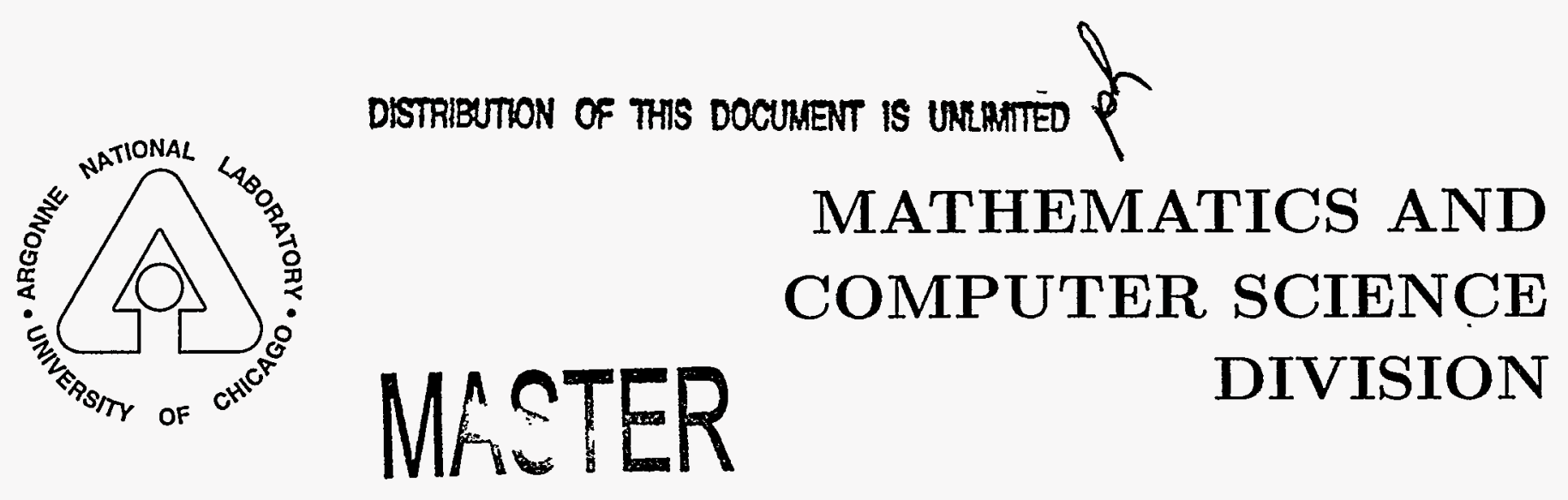


Argonne National Laboratory, with facilities in the states of Illinois and Idaho, is owned by the United States government, and operated by The University of Chicago under the provisions of a contract with the Department of Energy.

\section{DISCLAIMER}

This report was prepared as an account of work sponsored by an agency of the United States Government. Neither the United States Government nor any agency thereof, nor any of their employees, makes any warranty, express or implied, or assumes any legal liability or responsibility for the accuracy, completeness, or usefulness of any information, apparatus, product, or process disclosed, or represents that its use would not infringe privately owned rights. Reference herein to any specific commercial product, process, or service by trade name, trademark, manufacturer, or otherwise, does not necessarily constitute or imply its endorsement, recommendation, or favoring by the United States Government or any agency thereof. The views and opinions of authors expressed herein do not necessarily state or reflect those of the United States Government or any agency thereof.

Reproduced from the best available copy.

Available to DOE and DOE contractors from the Office of Scientific and Technical Information

$$
\text { P.O. Box } 62
$$

Oak Ridge, TN 37831

Prices available from (423) 576-8401

Available to the public from the National Technical Information Service

U.S. Department of Commerce 5285 Port Royal Road Springfield, VA 22161 


\title{
ARGONNE NATIONAL LABORATORY \\ 9700 South Cass Avenue
}

Argonne, IL 60439

ANL/MCS-TM-216

\section{MolView Users Guide}

\author{
by \\ Brian P. Walenz
}

\begin{abstract}
Mathematics and Computer Science Division
\end{abstract}
Technical Memorandum No. 216

June 1996

This work was supported by the Mathematical, Information, and Computational Sciences Division subprogram of the Office of Computational and Technology Research, U.S. Department of Energy, under Contract W-31-109-Eng-38, and by the Argonne Director's Individual Investigator Program. 


\section{DISCLAIMER}

Portions of this document may be illegible in electronic image products. Images are produced from the best available original document. 


\section{Contents}

$\begin{array}{lll}\text { Abstract } & 1\end{array}$

1 Introduction . 1

2 Execution 2

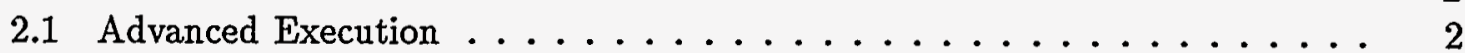

$2.1 .1 \mathrm{MPICH}, \mathrm{p} 4 \mathrm{Device} \ldots \ldots \ldots \ldots \ldots$

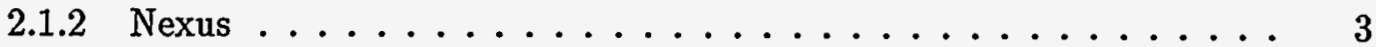

3 Driver Programs 5

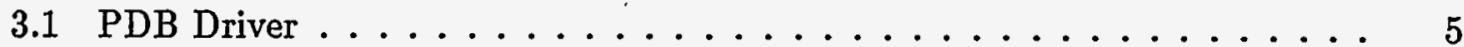

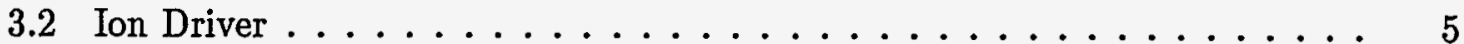

4 The CAVE Interface 7

4.1 Main Menu . . . . . . . . . . . . . . . . . . . 7

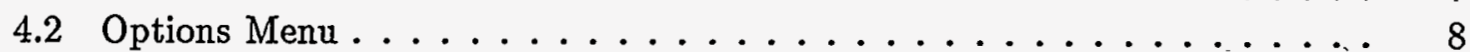

4.3 Frame Menu ......................... 8

5 For the Programmer 9

5.1 Directory Structure . . . . . . . . . . . . . . . . . . . 9

5.2 Building MolView ........................... 10

6 Driver Responsibility and Creation 10

6.1 Driver Initialization $\ldots \ldots \ldots \ldots \ldots \ldots \ldots$

6.2 Data Formats . . . . . . . . . . . . . . . . . . . . 11

6.3 Data Transmission . . . . . . . . . . . . . . . . . . . 12

6.3 .1 MV_AddMolecule ()$\ldots \ldots \ldots \ldots \ldots \ldots$

6.3 .2 MV_AddFrame ()$\ldots \ldots \ldots \ldots \ldots$

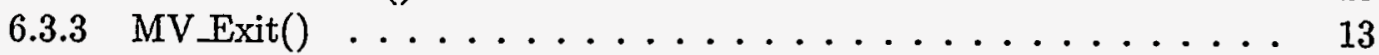

7 CAVE Program Modification $\quad 13$

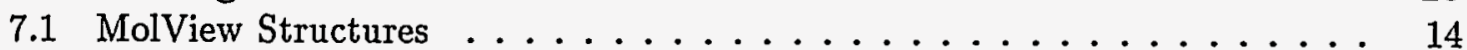

7.2 MPI Datatypes . . . . . . . . . . . . . . . . . . . . . 15

7.3 Driver to CAVE Transfer Functions . . . . . . . . . . . . . 15

7.4 CAVE Receive Functions . . . . . . . . . . . . . . 15

7.4.1 ReceiveMolecule ()$\ldots \ldots \ldots \ldots \ldots$

7.4 .2 ReceiveFrame ()$\ldots \ldots \ldots \ldots 16 \ldots \ldots$

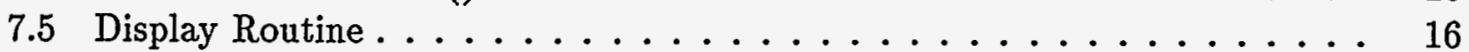

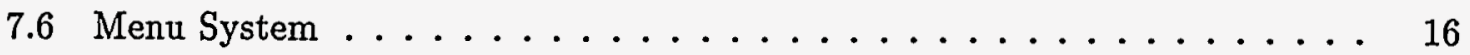

$\begin{array}{ll}\text { References } & 16\end{array}$

iii 


\title{
MolView Users Guide
}

\author{
by \\ Brian P. Walenz
}

\begin{abstract}
A system for viewing molecular data in a CAVE virtual reality environment is presented. The system, called MolView, consists of a frontend driver program that prepares the data and a backend CAVE program that displays the data. Both are written so that modifications and extensions are relatively easy to accomplish.
\end{abstract}

\section{Introduction}

MolView is an extensible system for viewing molecular data in the CAVE virtual reality environment. The user can view the molecule as if it were a physical model, rotating and viewing the object from different angles.

The MolView system consists of a backend CAVE program, and several routines that allow a frontend driver program to easily send data to the CAVE program. All communication is done via MPI, allowing the driver to be executed on a wide variety of machines.

MolView is capable of storing and displaying an arbitrary number of datasets, each of which may have an arbitrary number of time steps, or frames. Thus, there are four general types of data that can be viewed:

- Single dataset, single time step

- Single dataset, multiple time steps

- Multiple datasets, single time step per dataset

- Multiple datasets, multiple time steps per dataset

MolView can currently only display data. There are no mechanisms to allow the CAVE user to modify a dataset and send it back to the driver program for later computation.

The remainder of this document examines the execution of MolView applications (Section 2), two example driver programs, ion and $p d b$ (Section 3), and the CAVE interface (Section 4).

Section 5 introduces the programming level details of MolView. Sections 6 and 7 explain how to create custom drivers and modify the CAVE program to accept new data from custom drivers 


\section{Execution}

It is the responsibility of the driver program to supply the CAVE with data to show. How a driver generates the data is arbitrary; some drivers simply read a data file, whereas others compute the solution to a problem in real time.

For drivers that execute on a machine that the display trusts * execution is straightforward:

\section{MolView driver options [...] displayname}

Here, driver is a path to the driver executable that is to be used, options are the options to the driver, and displayname is the name of the display that the CAVE program is executed on, chosen from one of four forms, as shown below:

\begin{tabular}{|ll|}
\hline Display Type & Action \\
\hline local & Displays on the local monitor, equivalent to using ":0.0" \\
machine:display.screen & Displays on a remote monitor \\
cave & Starts the CAVE \\
idesk & Starts the ImmersaDesk \\
\hline
\end{tabular}

For example, to run the pdb driver program in bin to show all the pdb files in $/$ proteins and display them on the display attached to vogon, cd to the MolView home directory and type

\section{MolView bin/pdb \%proteins/* vogon:0.0}

The display type cave will perform all the magic that is necessary to start MolView in the CAVE; check first that the CAVE is free for use. The display type idesk will start MolView and show the display on the ImmersaDesk; you must ensure that the ImmersaDesk is free and take the projector out of standby mode. ${ }^{\dagger}$ Both cave and idesk are Argonne specific. See the MolView script itself for low-level execution details.

\subsection{Advanced Execution}

When the driver is executed on a machine that is not trusted, or is a parallel program, the Molview script will not work. The first problem is solved by making the machine trusted or by using a secure server. Consult your support staff or a local MPI guru for help.

\footnotetext{
*An easy way to tell if machine $A$ trusts machine $B$ is to attempt a remote shell from machine $B$ to machine A, for example, rsh A date.

"Point the projector remote control at the ImmersaDesk screen and press and hold the "standby" button. Repeat to turn off-just be sure to hold the button down!
} 
local

spnode2

spnode 3

spnode4

spnode5

alaska.mes.anl.gov

\section{0}

1 /sphome/walenz/ION/ion. $x$

1 /sphome/walenz/IoN/ion. $x$

1 /sphome/walenz/ION/ion. $x$

1 /sphome/walenz/ION/ion. $x$

1 /afs/fl/home/walenz/ION/bin/StartMolViewCave

Figure 2.1: Sample procgroup file using $5 \mathrm{SP}$ nodes and showing the results in the CAVE

When the driver is executed in parallel on a trusted machine, startup will need to be handled explicitly. This process depends heavily on which communication device is being used, and that depends on which machine the driver is being run on. The next two sections explain two common methods at Argonne.

\subsubsection{MPICH, p4 Device}

The MPICH communication method exists for most machines and is relatively easy to use. First, a procgroup file must be created where the driver is executed. This file contains a list of machines and the program they should run. A sample procgroup file is shown in Figure 2.1. The first column contains the name of the machine, the second is the number of processes to run on this machine, and the last is the executable to run. The first entry, local 0 says to run 0 additional copies on the current machine.

The $\mathrm{p} 4$ device uses Unix sockets to perform the actual communication. This strategy allows it to run on a wide variety of machines, but also means that performance is not optimal.

Once the procgroup file is created, the application is launched with driver -p4pg procgroup. Note that this will fail if the machines listed do not trust the local machine, usually returning permission denied.

\subsubsection{Nexus}

For better performance when using a multiprocessor driver on the IBM SP, Nexus MPI may be used. Instead of using $\mathrm{p} 4$ for all communication, Nexus uses MPL for communication between SP nodes, and $\mathrm{p} 4$ between the SP and the CAVE.

Like MPICH p4, Nexus needs a list of what to run. Since two different methods of communication are being used (MPL and p4), two different lists of processors are needed. Jobs started using the MPL startup routines are specified in much the same way that normal MPL jobs are specified; the user defines a set of envrionment variables.

For machines that are not started via the MPL startup routines, Nexus consults a database file (Figure 2.2). Like the p4 procgroup, this file tells Nexus what executable 


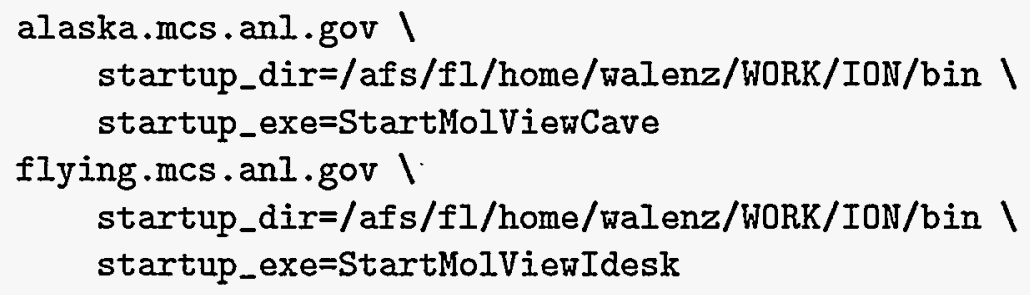

Figure 2.2: Sample Nexus database file

to run on various machines. The example in Figure 2.2 has two entries: the CAVE on alaska.mcs.anl.gov, and the ImmersaDesk on flying.mcs.anl.gov. Which one is used depends on the command line used to start the jobs.

$\# ! / \mathrm{bin} / \mathrm{sh}$

MP_HOSTFILE=/sphome/\$LOGNAME/SPnodes . 'get jid'

MP_PROCS='cat \$MP_HOSTFILE I WC -1 '

MP_PULSE $=0$

MP_EUILIB=us

export MP_HOSTFILE MP_PROCS MP_PULSE MP_EUILIB

ion.x -mpi -dbfile $/$ SP/demo.rdb -nodes alaska.mcs.anl.gov -nonameexpand

Figure 2.3: Sample Nexus startup script

The script in Figure 2.3 will start the multiprocessor driver on the SP and the CAVE on alaska.mcs.anl.gov.

The magic behind Nexus startup is in the command line ("ion. $x$-mpi ..."):

- -mpi tells Nexus that the remaining arguments are for it.

- The Nexus database file is/sphome/walenz/SP/demo.rdb.

- In addition to the MPL job startup, start a job on alaska.mcs.anl.gov. The parameters for the job are defined in the database file.

- Do not use the name ion. $x$ when starting the alaska.mcs.anl.gov job. 


\section{Driver Programs}

\subsection{PDB Driver}

The PDB driver pdb will accept several input .pdb files, which can be compressed (names ending with ".gz" or ".Z") or uncompressed (names ending with anything else). The program will parse the file and send a list of atoms to the CAVE that are sized and colored as follows:

\begin{tabular}{|lll|}
\hline Atom Type & Color & Relative Size \\
\hline Nitrogen & Blue & 0.58 \\
Hydrogen & White & 0.58 \\
Carbon & Green & 0.73 \\
Sulfur & Yellow & 0.90 \\
Oxygen & Red & 0.50 \\
Unknown & Dark Gray & 0.25 \\
\hline
\end{tabular}

To change these values modify the pdb driver code and recompile.

Note: No bonds are shown between atoms.

Because of speed considerations, only the first 2000 atoms are used. If more than 2000 atoms are present in a given file, a warning message is printed, and the rest are ignored. This approach is taken because showing large numbers of atoms is exceptionally slow.

The pdb driver accepts any number of command line arguments. All of them are treated as names of .pdb files to show.

\subsection{Ion Driver}

The ion driver ion will read in a data file of ion positions, process the data to determine a shell structure, color each shell differently, and send this processed molecule to the CAVE.

The ion driver allows all four types of execution described in the introduction.

The general format of the data file is shown in Figure 3.1, and a sample input file is in Figure 3.2.

The ion driver has one mandatory command line argument, the name of the data file to show. An optional argument, -d, forces ion to use a distance-based coloring scheme rather than the default shell-based scheme, and is useful for viewing data sets with more than one frame that do not have a shell structure-the visualization of a minimization procedure, for example. It prevents, (or tries to prevent) the color scheme from changing rapidly while viewing the minimization process. In some cases, it will come close to coloring each shell differently; in others, a single shell might have two different colors. The - $\mathrm{d}$ argument must be the first argument on the ion command line. 


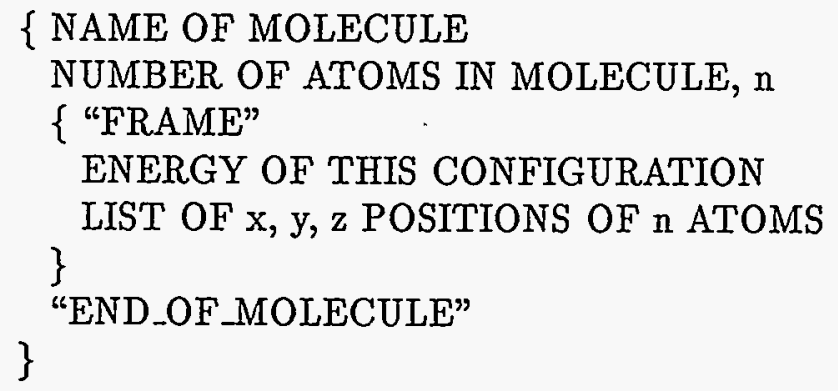

Figure 3.1: Generai ion input file format. The brackets represent blocks in the input file. Each block must be included at least once. There is no limit on the number of blocks.

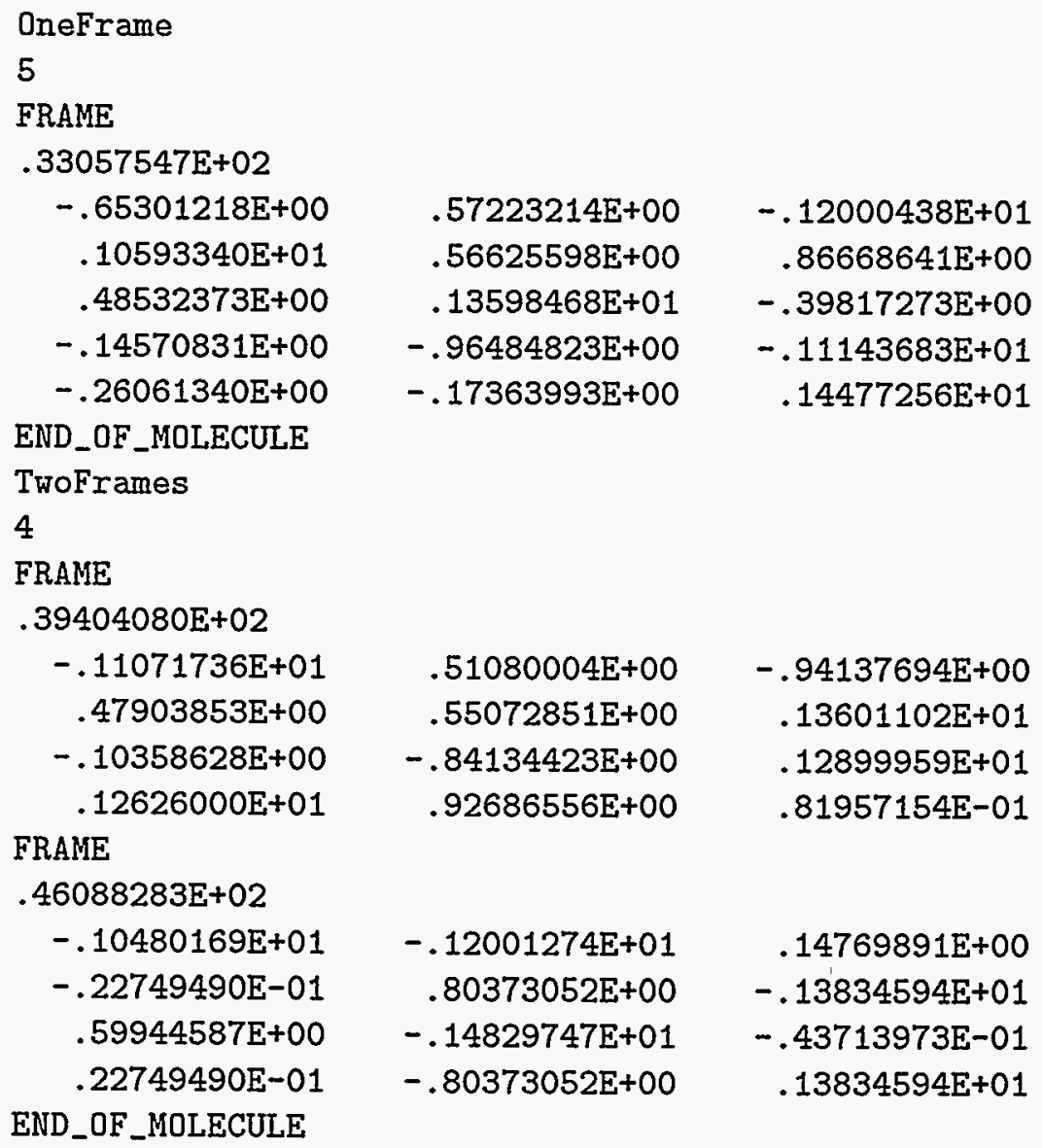

$$
\begin{array}{rr}
.57223214 \mathrm{E}+00 & -.12000438 \mathrm{E}+01 \\
.56625598 \mathrm{E}+00 & .86668641 \mathrm{E}+00 \\
.13598468 \mathrm{E}+01 & -.39817273 \mathrm{E}+00 \\
-.96484823 \mathrm{E}+00 & -.11143683 \mathrm{E}+01 \\
-.17363993 \mathrm{E}+00 & .14477256 \mathrm{E}+01
\end{array}
$$

$$
\begin{array}{rr}
-.12001274 \mathrm{E}+01 & .14769891 \mathrm{E}+00 \\
.80373052 \mathrm{E}+00 & -.13834594 \mathrm{E}+01 \\
-.14829747 \mathrm{E}+01 & -.43713973 \mathrm{E}-01 \\
-.80373052 \mathrm{E}+00 & .13834594 \mathrm{E}+01
\end{array}
$$

Figure 3.2: Sample data file for the ion driver 


\section{The CAVE Interface}

MolView uses the CaveMenu system [4]. Briefly, interaction with the menus is done by pointing the wand at a menu gadget and pressing the third (right) button.

The menus in MolView are grouped by function. Each menu is callable from the main menu by selecting the appropriate button.

\subsection{Main Menu}

The main menu (Figure 4.1) consists of a few buttons to call other menus (options, frame), buttons to toggle modes (rotation, translation), a slider to change what data set is shown, and the "Quit" button.

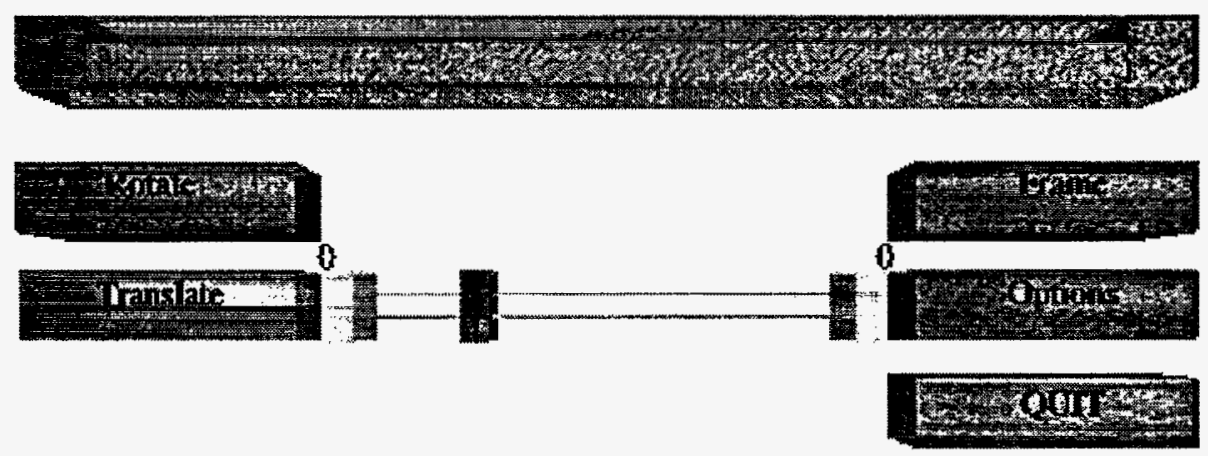

Figure 4.1: The main menu

Selecting Options or Frame will bring up the options menu (Section 4.2) or frame menu (Section 4.3), respectively.

Rotate and Translate toggle the ability to rotate and translate the molecule in the CAVE. When Rotate is selected, pressing the left wand button and rotating the wand will rotate the molecule. If the left button is released while the wand is still rotating, the molecule will continue to rotate at a constant rate. To stop the molecule from rotating, either hold the wand steady and release the left button, or turn off the Rotate menu button. When Translate is selected, holding the middle wand button and moving the wand will move the molecule in the CAVE.

The main menu also contains a slider gadget that allows you to select the dataset to view. Clicking on the slider knob allows you to drag the knob, while clicking on the slider beams will snap the slider knob to that position. Clicking on the ends of the slider bars will move the knob in that direction one step.

Selecting Quit will quit the application. 


\subsection{Options Menu}

The options menu (Figure 4.2) has three sliders. From the top, there are sliders to change the detail level of atoms, size of atoms, and size of the molecule.

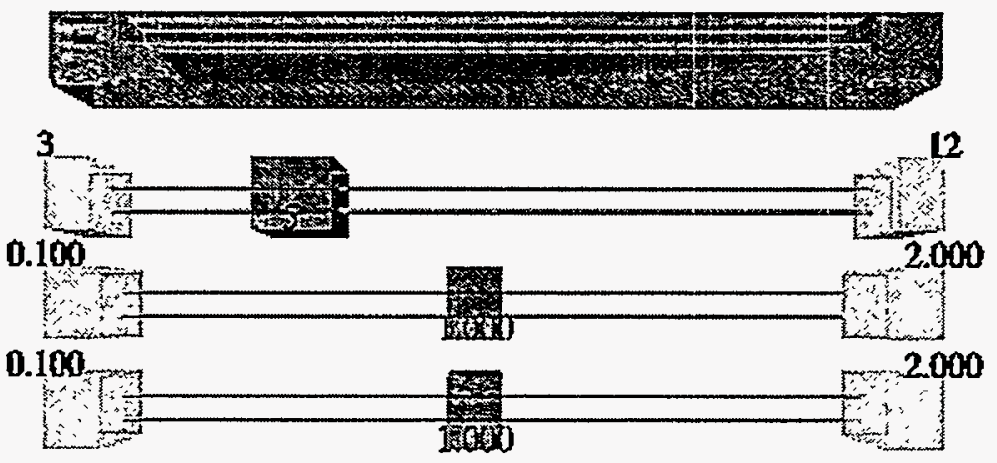

Figure 4.2: The options menu

The top slider controls how smooth the atoms appear. By increasing the detail, more polygons are used to draw each sphere. Be forewarned; beauty comes at a large price. The CAVE can draw only a finite number of polygons.per second, so increasing the detail level will directly affect the response time. The result might not be so terrible when using the CAVE simulator, but when in the CAVE every move will (usually) change the viewing angle. If the response time is very high, the CAVE will appear to be jumpy; if the response time is low, the CAVE can smoothly keep up with your movements, giving a more realistic three-dimensional illusion.

The middle slider changes the size of all atoms in the molecule. This can be used to transform the molecule to a space-filling model.

The bottom slider scales the molecule, either bringing the atoms closer together or spreading them farther apart. This is useful for making large molecules manageable and small molecules large enough to see. Note that changing the size of the molecule not change the size of the atoms.

\subsection{Frame Menu}

For molecules with more than one frame, the frame menu (Figure 4.3) allows you to cycle through all the frames in the dataset.

Two methods exist for viewing other frames: using the slider, or clicking on Reverse or Forward. By using the slider, you can quickly view the entire sequence of frames, but using Reverse or Forward will iterate through the sequence of frames like a movie. Toggling Cycle will let Forward and Reverse loop from end to end.

Faster and Slower change the speed that Reverse and Forward iterate through the frames: 


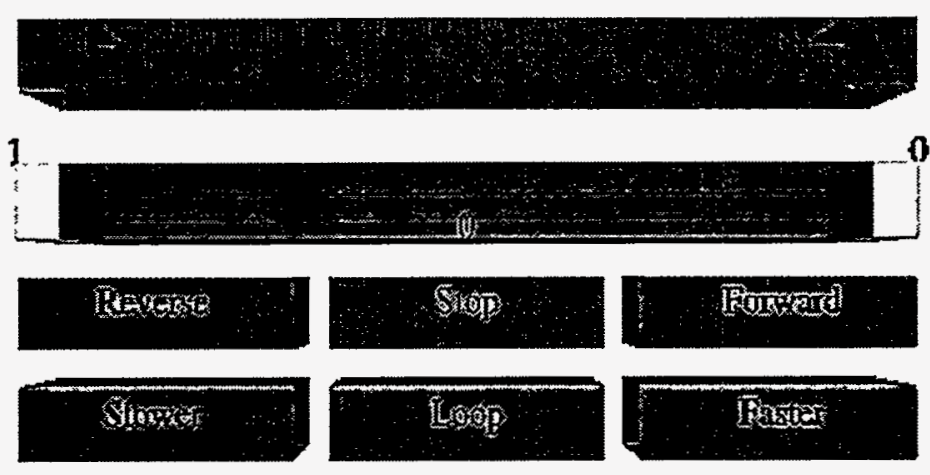

Figure 4.3: The frame menu

To stop playback, either turn off Reverse or Forward (whichever is on), or select Stop.

\section{For the Programmer}

The remainder of this document describes MolView from a programmer's perspective. First, the directory structure and compilation of MolView are explained. Next, driver responsibilities and driver creation are discussed. Finally, the internals of the CAVE component of MolView and techniques for extending MolView to show different styles of data are described.

\subsection{Directory Structure}

The directory structure is simple:

\begin{tabular}{|l|l|}
\hline bin & Binaries and the MolView execution script \\
drivers & Driver source code \\
include & Header files \\
lib & Driver library files-libMolView.a \\
obj & Object code from building MolView \\
src & MolView CAVE and library source code \\
\hline
\end{tabular}

In include are the following:

\begin{tabular}{|l|l|}
\hline MolView.h & Datatype definitions \\
MolViewCave.h & CAVE internal datatypes \\
\hline
\end{tabular}


In src are the following:

\begin{tabular}{|l|l|}
\hline CleverMath.C & Some mathematics \\
Datatypes.c & Handles the creation of MPI datatypes for sending and receiving data \\
Display.C & The CAVE draw routine \\
DisplayList.C & User display list creation function \\
Initialize.C & Initialization and memory handing routines \\
Menus.C & Menu creation and handling \\
Receive.C & Routines for receiving data from driver programs \\
Remote.c & Routines for sending data from drivers to the CAVE \\
Cave.C & Main CAVE routine \\
\hline
\end{tabular}

\subsection{Building MolView}

To build MolView, one simply types make from the MolView root directory. This will build MolViewCave in bin/, libMolView.a in $1 \mathrm{ib} /$, and any drivers in drivers/. If the build is being done on a multiprocessor machine, a parallel build will be done if the PARALLEL environment variable is set to the number of steps to perform concurrently.

The command make clean will remove the object files, and make distclean will remove everything that is generated by make, including libraries and executables.

\section{Driver Responsibility and Creation}

Driver programs are responsible for one important task-creating data for the CAVE to show. This task comprises three pieces, although the line between each piece is fuzzy.

1. Create the data. This task can be done by the driver program, for example, by embedding the MolView system into a prewritten computational program. Alternatively, the driver program can just read a data file.

2. Massage the data into the MolView data structures (Section 6.2).

3. Transfer the data to the CAVE (Section 6.3).

These do not need to be distinct steps and, in some cases, must not be distinct. The simplest example here is a driver program that computes the data using MolView data structures: computation and packaging step are merged. An example merging all three steps is a driver that minimizes the energy of a molecule and sends the molecule to the CAVE after each minimization step. 


\subsection{Driver Initialization}

To ease the pain of creating a multiprocessor driver, every driver (even uniprocessor!) must call MV_SplitCommunicator() (or MVF_SplitCommunicator() if in Fortran) immediately after calling MPI Init(). This routine will create a new MPI communicator consisting of all the nodes that are driver nodes, allowing the driver to do collective communication between driver processors only.

Failure to call this routine will result in the CAVE locking up immediately after startup.

\subsection{Data Formats}

Three data structures exist for driver to CAVE communication: struct MoleculeData, struct FrameData, and struct AtomData, all of which are defined in include/MolView.h.

The struct MoleculeData data structure contains book-keeping information necessary to tell the CAVE about each molecule. MoleculeID is how a driver refers to a CAVE molecule and thus should be unique. The CAVE stores molecules in increasing MoleculeID order.

A description string is provided; however, its use is not mandatory. If a description is not needed, the string should the set to zero length.

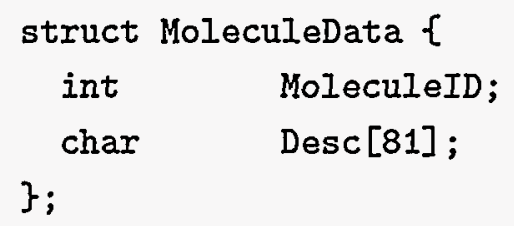

The struct FrameData data structure is an atom-level description of the molecule. Since frames are added independently of molecules, each frame needs both a FrameID and a MoleculeID. The FrameID allows the CAVE to order the frames, while the MoleculeID tells the CAVE which molecule this frame is a part of.

NumAtoms is the number of atoms that this frame contains. No atoms are stored in FrameData; instead, they are passed in separately to MV_AddFrame() (Section 6.3.2).

Center is the coordinate that the CAVE uses to rotate about. To have the molecule rotate about the center of mass, Center should be set the the center of mass. Likewise, to rotate about a specific atom, Center should be the location of that atom.

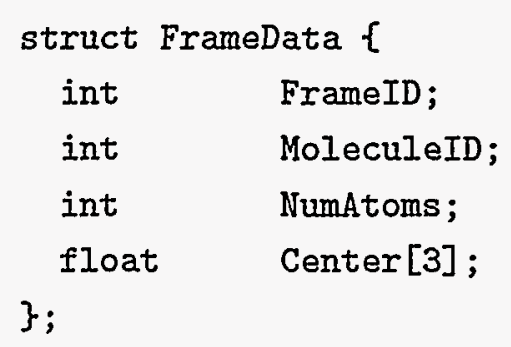




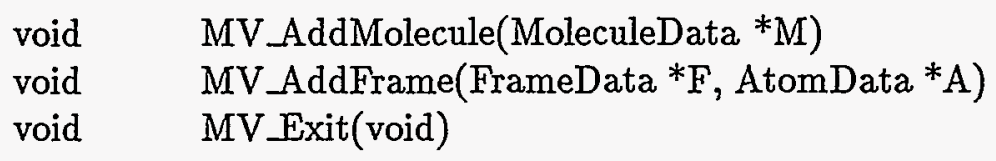

Figure 6.1: C driver to CAVE interface

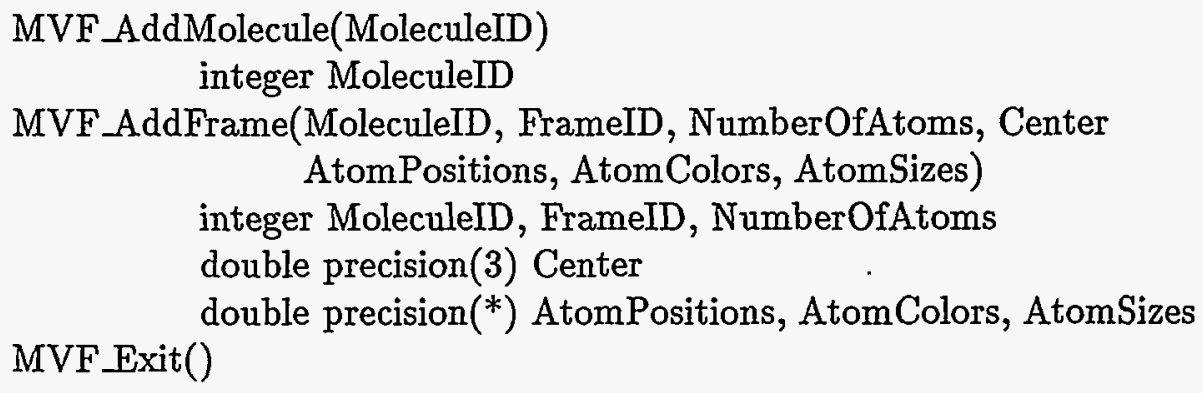

Figure 6.2: Fortran driver to CAVE interface

The AtomData data structure contains a complete description of a single atom in the molecule. At the very least, this description must contain the position, color and size of the atom.

struct AtomData \{

float Position[3];

float Color [4];

float Size;

\};

\subsection{Data Transmission}

A driver program uses three routines to communicate with the CAVE: one to create a molecule, one to add a frame to a previously created molecule, and one to tell the CAVE that the driver is done. Since Fortran is not able to use $\mathrm{C}$ structures, separate routines exist for $\mathrm{C}$ (Figure 6.1) and Fortran (Figure 6.2).

For low-level details, see the code in Remote.c and Datatypes.c, as well as Section 7. This section explains only how to use the routines in a driver program and assumes that the required data structures are completely and correctly created.

Several sample drivers are provided in drivers/; simpledriver.c, framedriver.c, and simplefort.f. 


\subsubsection{MV_AddMolecule()}

MV_AddMolecule() requests that the CAVE allocate space for a new molecule with ID MoleculeID. No frames are transmitted or allocated.

From Fortran, it is not possible to pass a description string to the CAVE.

\subsubsection{MV_AddFrame()}

MV_AddFrame() adds a frame to the molecule with ID MoleculeID. If the molecule has not been created, the CAVE will print an error message and fail.

The atom list does not need to be sorted, but several optimizations can be made if it is. See the code in DisplayList.C for ideas.

The choice of double precision in the Fortran interface is reasonably arbitrary. Look in Remote.c for details on what needs to be changed to use real.

\subsubsection{Exit()}

This routine should be called when the driver is done sending data to the CAVE. Once called, the driver may proceed with any cleanup it needs to do, then call MPI Finalize(), and exit. The CAVE will continue to allow the user to view the data.

\section{CAVE Program Modification}

The structure of the CAVE portion of MolView roughly consists of two parts: initialization and communication. Everything else is handled through callback routines by the CaveMenu or CAVE library.

Them main loop (in cave.C) performs several initialization tasks:

- Starts MPI, and creates a driver-only communicator.

- Allocates a large chunk of shared memory for the menus and MolView data.

- Initializes the basic MolView data structures.

- Creates the menus.

- Configures the CAVE, and sets the callback routines.

Once initialized, MolView enters a loop where it receives data from the driver until either the ESC key is pressed or the driver announces it is done.

The behavior of MolView is changed by modifying the MolView data structures and then modifying the various callback routines that act on the data structures. The callback routines that need to be modified are in: 
Datatypes.c MPI datatypes.

Remote.c Driver to CAVE transfer functions.

Receive.C CAVE receive functions.

Display.C CAVE display list generation routine.

Menus.C The Menu system.

Such modifications, even for moderate extensions, are not excessively involved. As an example, look through the ION code. All the "shell" operations are essentially an extension to the base MolView system.

Note: Referring to the code while reading this section will greatly enhance comprehension.

\subsection{MolView Structures}

The MolView structures specific to the CAVE contain an instance of the appropriate driver structure (CAVEAtom contains AtomData, for example) and any additional storage that the CAVE needs.

CAVEAtom currently does not contain any additional information. Later, for example, if one wishes to draw sticks to connect atoms, an array of pointers to other CAVEAtoms could be added.

Note that if the AtomData data structure is modified, ReceiveFrame() must be modified as well. See Section 7.4.2 for an explanation.

struct CAVEAtom \{

AtomData Data;

\};

CAVEFrame contains a list of the atoms in this frame and a pointer to the next frame in the sequence.

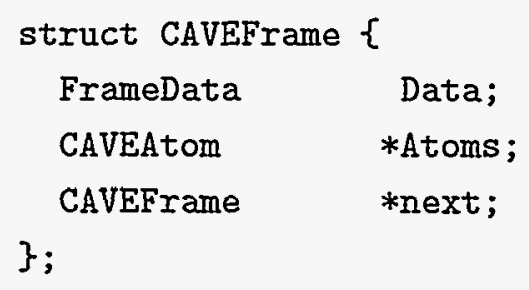

NumFrames, CurrentFrame, $\mathrm{cF}$, and Frames are used to keep track of which frame is currently being shown, and should be included in all derived data structures.

struct CAVEMolecule \{ 


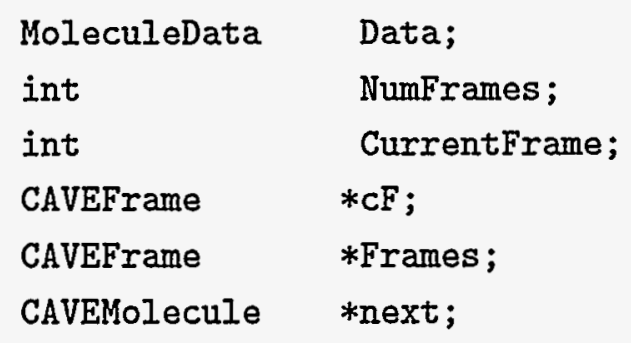

\subsection{MPI Datatypes}

The routines in Datatypes.c are responsible for informing MPI about the data that one intends to send. These routines are critical. If they are incorrect, MPI will (probably) send junk data to the CAVE.

Basically, MD_Create*Datatype() determines the size and relative position of every block of data. A block of data is any number of structures that all have the same type, so four int variables in a row is one block.

When modifying the MolView structures, one simply modifies MD_Create*Datatype() so that the blocks of data are specified. For more information, see an MPI manual such as $[1,2]$.

\subsection{Driver to CAVE Transfer Functions}

The driver to CAVE transfer functions are in Remote.c. They create the MPI datatype(s) needed and call MPI_Send(). All the work is done by the MPI datatype. These functions probably will not need to be modified.

\subsection{CAVE Receive Functions}

The CAVE receive functions in Receive.C are responsible for receiving the data from a driver and placing it in the CAVE data structures. While doing this, they could optimize the data, for example, taking array indices and translating them to $\mathrm{C}$ pointers.

\subsubsection{ReceiveMolecule()}

The molecule receive function should need very little attention. The molecule data structure is responsible for holding all the CAVE related book-keeping information. Any user-defined data generally is held in struct MoleculeData, which is received directly to the CAVE data structure. 


\subsubsection{ReceiveFrame()}

The frame receive function is not nearly as friendly as the molecule receive function. Atoms are stored in the frames as an array. CAVEFrame contains an array of CAVEAtom to allow for CAVE local data in an atom (a list of pointers to other atoms, for example). Since CAVEAtom and AtomData generally will be different, atom data must be copied manually to the array in CAVEFrame. Thus, any changes to struct CAVEAtom must be propagated through to ReceiveFrame().

\subsection{Display Routine}

Modification of the display routine should consist just of changing the display list creation routine, ComputeDisplayList(), in DisplayList.C. Anything that is legal in an OpenGL display list is legal here, but everything that is done here will be treated as part of the molecule-they will rotate and translate with the molecule. If the behavior of the molecule needs to be modified, then Draw () in Display. $($ will need to be modified.

Note: since each processor must have its own local copy of the display list, each processor must call ComputeMoleculeDisplayList() whenever the molecule changes. Failure to do so will result in the correct molecule being displayed on one CAVE wall, and something else on other CAVE walls.

See the OpenGL Programming Guide [3] for details on display list creation.

\subsection{Menu System}

Menu routines are in Menus. $C$ and can be modified according to [4].

Sliders merit special attention: they must be updated whenever the values they are associated with change. Currently, they are updated whenever something happens, not just when their value changes. Ambitious programmers could figure out when the sliders must be updated, and only update there, but most programmers will realize that the overhead for updating constantly is low.

Also meriting attention is RemakeMolecule(). This function tells the display processes to create a new display list, in addition to updating sliders.

\section{References}

[1] M. P. I. Forum, MPI: A message-passing interface standard, International Journal of Supercomputing Applications, 8 (1994).

[2] W. Gropp, E. Lusk, ANd A. Skjellum, USING MPI Portable Parallel Programming with the Message-Passing Interface, The MIT Press, Cambridge, 1994. 
[3] M. W. J. Neider, T. Davis, OpenGL Programming Guide, Addison-Wesley Publishing Company, Reading, Massachusetts, 1993.

[4] B. Walenz. Unpublished information, Argonne National Laboratory, 1996.

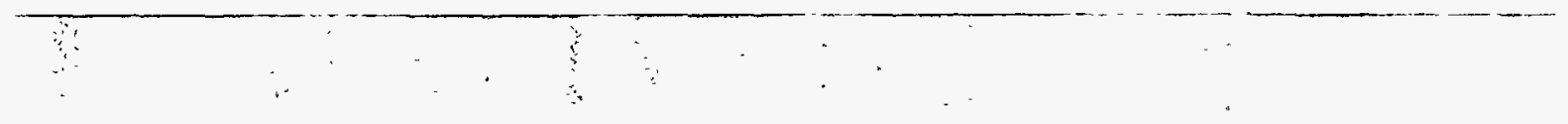

